\title{
Mechanical force sensors using organic thin-film transistors
}

\author{
Grzegorz Darlinski, ${ }^{\text {a) }}$ Ulrich Böttger, and Rainer Waser \\ Institute of Materials in Electrical Engineering and Information Technology 2 (IWE 2), \\ Rheinisch-Westfalische Technische Hochschule Aachen (RWTH)-Aachen University, \\ D-52074 Aachen, Germany
}

Hagen Klauk, Marcus Halik, Ute Zschieschang, Günter Schmid, and Christine Dehm New Memory Platforms, Materials and Technology, Infineon Technologies, Paul-Gossen-Straße 100, 91052 Erlangen, Germany

(Received 26 October 2004; accepted 21 February 2005; published online 19 April 2005)

\begin{abstract}
The pressure dependence of pentacene $\left(\mathrm{C}_{22} \mathrm{H}_{14}\right)$ transistors with solution-processed polyvinylphenol gate dielectric on glass substrates is investigated by applying uniaxial mechanical pressure with a needle. We found that organic thin-film transistors are sensitive to applied pressure inherently. The measurements reveal a reversible current dependence of the transfer characteristics where the drain current is switching between two states. Experimental and simulation results suggest that switch-on voltage and interface resistance are affected. The change takes seconds, hinting at trap states being responsible for the effect. (C) 2005 American Institute of Physics. [DOI: 10.1063/1.1888046]
\end{abstract}

\section{INTRODUCTION}

Organic semiconductors are of great interest for lowcost, large-area electronics. Applications such as information displays, chemical sensors, humidity sensors, and electronic paper have been implemented. ${ }^{1-4}$ Pentacene organic thin-film transistors (OTFTs) show carrier mobility of up to $3.2 \mathrm{~cm}^{2} / \mathrm{V} \mathrm{s}$ and on/off current ratio exceeding $10^{6} .^{5}$ This is competitive to amorphous silicon transistors, which are used in liquid-crystal displays and flat-panel image detectors. But organic semiconductor circuits can be fabricated on flexible substrates and even on paper since the processing temperature is lower than for amorphous silicon-an advantage that could grant access to future applications. Printing techniques promise fast and easy fabrication. ${ }^{6,7}$ Recently improved dielectrics have enabled low-voltage organic devices. ${ }^{8}$

Someya and Sakurai demonstrated an artificial skin based on organic TFTs and rubbery pressure sensors. ${ }^{9}$ A flexible low-cost large-area sensor matrix enables robots with sense of touch. In this paper, we present the basics of a concept which is based solely on organic TFTs as the sensing element, without the need of any additional sensor element. Our approach would greatly simplify the fabrication because only TFTs are required eliminating all further process steps such as rubber deposition and additional structuring.

\section{EXPERIMENTAL PROCEDURES}

The thin-film transistors studied here consist of 30-nm-thick sputtered titanium gates on a glass substrate, covered with a 200-nm-thick polyvinylphenol (PVP) gate dielectric. Source and drain contacts are made of 30-nm-thick evaporated gold. The organic active layer is deposited by thermally evaporating pentacene in vacuum, with a thickness of about $30 \mathrm{~nm}$. The devices are covered with polyvinyl alcohol (PVA) for passivation [see Figs. 1(b) and 2(b)]. All functional layers are patterned by photolithography and etch-

${ }^{a)}$ Electronic mail: darlinski@iwe.rwth-aachen.de ing. A more detailed description of the production process is given elsewhere. ${ }^{10,11}$ Two distinct transistor layouts were evaluated in this work: TFTs with interdigitated source/drain contacts and relatively short channels (channel length $10 \mu \mathrm{m}$, channel width $1000 \mu \mathrm{m}$ ), and TFTs with a standard (linear) contact arrangement and relatively long channels [channel length $100 \mu \mathrm{m}$, channel width $100 \mu \mathrm{m}$; see Figs. 1(a) and 2(a)].

To study the pressure dependence of the electrical performance of these devices we apply mechanical force directly to the TFTs using a microneedle (see Fig. 3). We use a tungsten needle that is coated with a thick layer of a polymer photoresist to eliminate the possibility of short-circuiting the source and drain contacts of the transistors. The needle is attached to a fitting, and moved by a step motor. The TFT substrate is placed on a single-pan balance so that the pressure can be measured. The electrical characterization of the TFTs with and without applied mechanical pressure is carried out with an HP 4155B semiconductor parameter analyzer.

\section{RESULTS AND DISCUSSION}

Figure 2(c) shows the transfer characteristics (drain current $I_{D}$ versus gate-source voltage $V_{\mathrm{GS}}$ ) of a TFT with interdigitated contacts (channel length $10 \mu \mathrm{m}$ ) with and without applied pressure. In this measurement pressure was applied to the entire device, i.e., to the pentacene channel and the source/drain contact regions. The time for one sweep was about $10 \mathrm{~s}$. The transfer characteristics show a large increase in drain current when pressure is applied. The increase in drain current is most significant near the turn-on voltage of the TFT, i.e., between about -10 and $+10 \mathrm{~V}$. The observed increase in drain current is reproducible and reversible as during the measurement the needle was dropped and lifted several times between the voltage sweeps.

We have also examined the TFTs with standard (linear) contacts (with a channel length of $100 \mu \mathrm{m}$ ) where the needle 
(a)
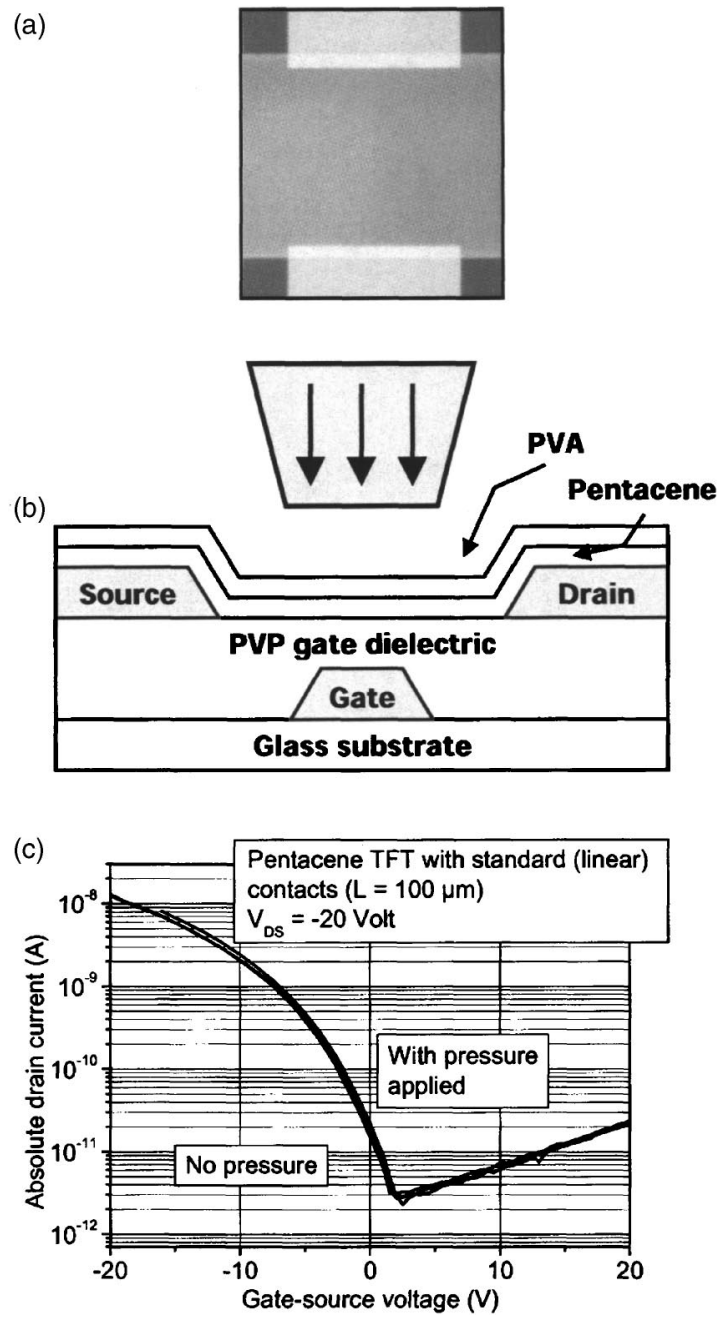

FIG. 1. (a) Optical micrograph of a pentacene TFT with standard (linear) contacts and a channel length of $100 \mu \mathrm{m}$; (b) schematic cross section of the experiment when pressure is applied only to the channel; (c) and transfer characteristic of a TFT with standard (linear) contacts and a channel length of $100 \mu \mathrm{m}$.

impacts only the pentacene channel, but not the contacts. These measurement did not show any change in drain current [see Fig. 1(c)].

The transfer characteristics shown in Figs. 1(c) and 2(c) were obtained with a pressure of about $10^{6} \mathrm{~Pa}$, calculated by dividing the force (measured with the balance) by the estimated tip area. Raising the pressure above $10^{6} \mathrm{~Pa}$ did not lead to any further changes in current.

One feasible mechanism for the observed effect could be that the increase in drain current is caused by leakage currents, either due to a short circuit through the tungsten needle or by crushing the dielectric (creating shorts to the gate electrode). To analyze this possibility we have measured all three TFT currents (drain current $I_{D}$, source current $I_{S}$, and gate current $I_{G}$ ) for both pressure states. The results [see Fig. 4(a)] indicate that, although leakage paths between the gate electrode and the source/drain contacts exist, the pressureinduced changes are entirely in the drain and source currents while the gate current is not affected by the mechanical pressure. We therefore conclude that the gate dielectric is not damaged by the needle and that the observed effect is not due to an increase in gate leakage. (a)

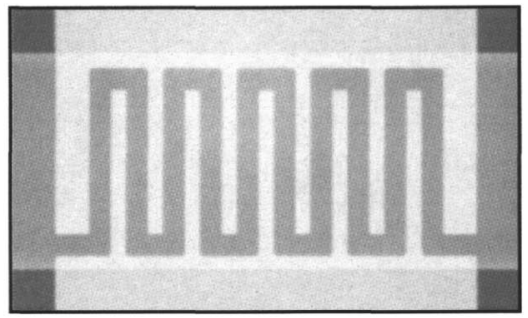

(b)
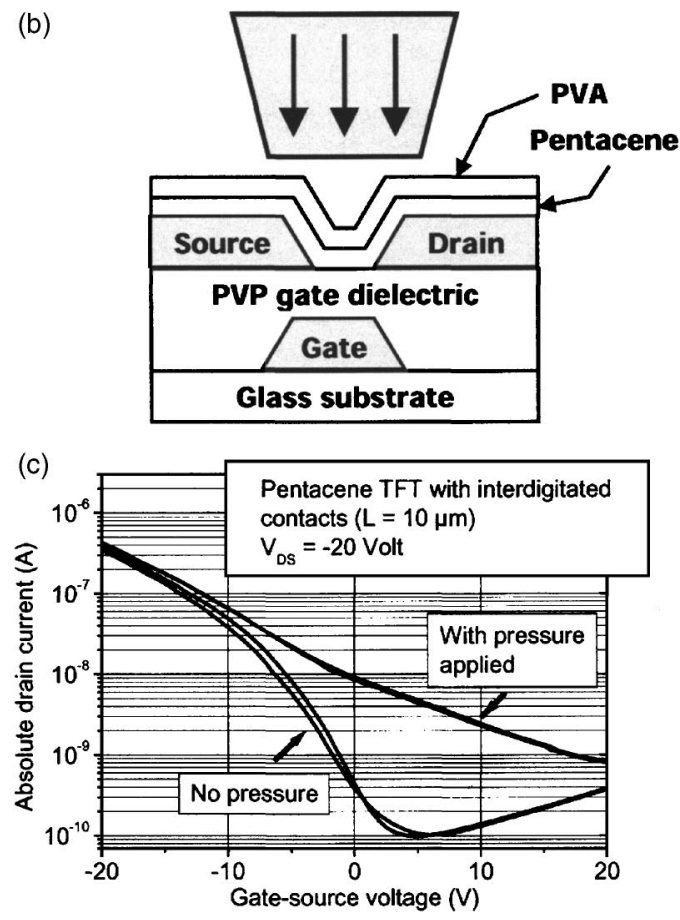

FIG. 2. (a) Optical micrograph of a pentacene TFT with interdigitated source/drain contacts and a channel length of $10 \mu \mathrm{m}$; (b) schematic cross section of the experiment when pressure is applied to the entire device, including the pentacene channel and the source/drain contact regions; (c) and transfer characteristic of a TFT with interdigitated source/drain contacts and a channel length of $10 \mu \mathrm{m}$, showing the pressure and no-pressure states.

Furthermore, the absolute and relative pressure-induced changes in the drain current shown in Fig. 4(b) indicate that the observed effect is not a leakage current through the tungsten needle, which would be expected to result in a constant current, i.e., a current independent of the gate-source voltage, which is obviously not the case. The data in Fig. 4(b) also show that the observed increase in drain current is not merely due to a pressure-induced reduction in gate dielectric thickness (which would cause an increase in the gate field $E$ for the same gate-source voltage $V_{\mathrm{GS}}$, since $E=V_{\mathrm{GS}} / t$, where $t$ is the dielectric thickness). If this was the case we would expect the relative change in drain current to be independent of the gate-source voltage, which is obviously not the case here. Therefore, we can rule out that the observed effect is due to elastic material effects or erroneous measurement setup.

While we see two stable states for slow measurements (i.e., measurements where the gate-source voltage is swept slowly between positive and negative values), fast measurements with total sweep times of about $200 \mathrm{~ms}$ show a smooth transition from the "low-pressure" state to the "highpressure" state [see Figs. 5(a) and 5(b)]. To examine the time dependence of the pressure effect we measure the drain cur- 


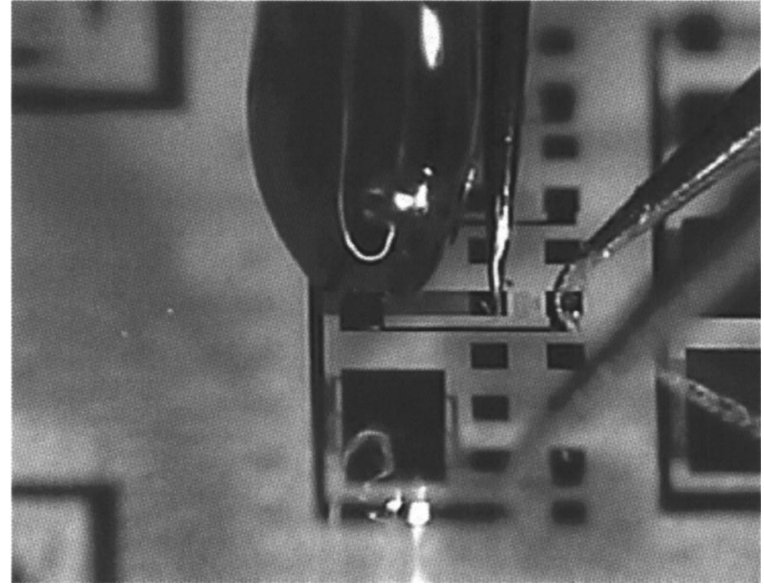

FIG. 3. Optical micrograph of needle positioned over a TFT with interdigitated source/drain contacts and a channel length of $10 \mu \mathrm{m}$.

rent at fixed gate and drain voltages selecting the voltages for maximum pressure sensitivity $\left(V_{\mathrm{GS}}=+10 \mathrm{~V}, V_{\mathrm{DS}}=-20 \mathrm{~V}\right)$, while moving the needle up and down [see Fig. 5(c)]. There was no measurable delay in response, but it takes about $20 \mathrm{~s}$ for the drain current to reach steady states. The rise and fall times (i.e., the time it takes for the current to change from $0 \%$ to $70 \%$ or from $100 \%$ to $30 \%$ ) are in the range of a few hundred milliseconds. The response appears to be much shorter when the current is not measured at fixed gate-source voltages but is swept between +10 and $-20 \mathrm{~V}$. Extending the waiting time at positive $V_{\mathrm{GS}}$ accelerates the transition. From this we conclude that the transition between the two steady states is mediated by trapping and detrapping of charges.

Trapped charges will influence a thin-film transistor in many ways, depending on the exact location of the traps in the device. From simply looking at the transfer characteristics it remains unclear which physical parameters of the TFT are influenced by the mechanical pressure. To further examine this we have used a SPICE program (simulation program with integrated circuit emphasis) to simulate the device. As discussed elsewhere ${ }^{12,13}$ the SPICE metal-oxidesemiconductor field-effect transistor (MOSFET) model is insufficient to simulate organic thin-film transistors. Therefore, we have modified the standard level-2 $p$-MOSFET model by introducing branches which form paths for leakage currents, as shown in Fig. 6. By adjusting the SPICE model parameters and improving the result by fitting the leakage currents we found a system which describes our device reasonably well. By changing MOSFET parameters we are able to simulate the transition from "pressure" state to "no-pressure" state with good accuracy. Figure 4(c) shows the currents calculated by SPICE. The agreement between experimental and simulation data is very good for positive gate-source voltages. For negative gate-source voltages the simulation is dominated by

$$
I_{D}=-K_{P}\left(\frac{W}{L_{\mathrm{eff}}}\right)\left(V_{\mathrm{GS}}-V_{\mathrm{TH}}-\frac{V_{\mathrm{DS}}}{2}\right) V_{\mathrm{DS}}\left(1-\lambda V_{\mathrm{DS}}\right),
$$

which describes the behavior of inorganic transistors in the linear regime, but which is inaccurate for organic devices. ${ }^{14}$ In Eq. (1) $I_{D}$ denotes the drain current for $0>V_{\mathrm{DS}}>\left(V_{\mathrm{GS}}\right.$

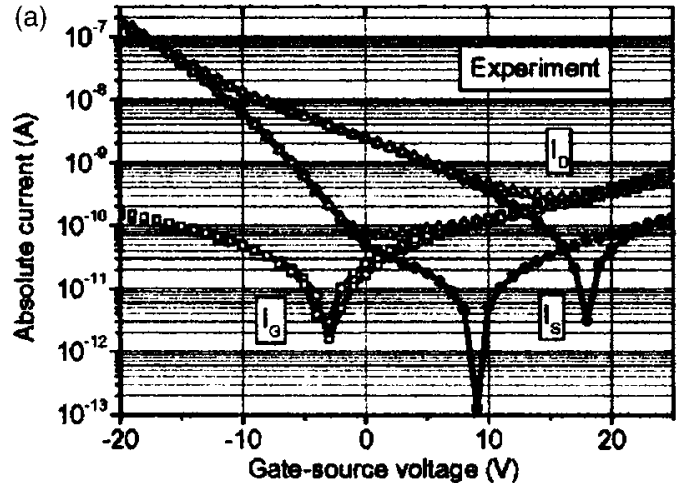

(b)
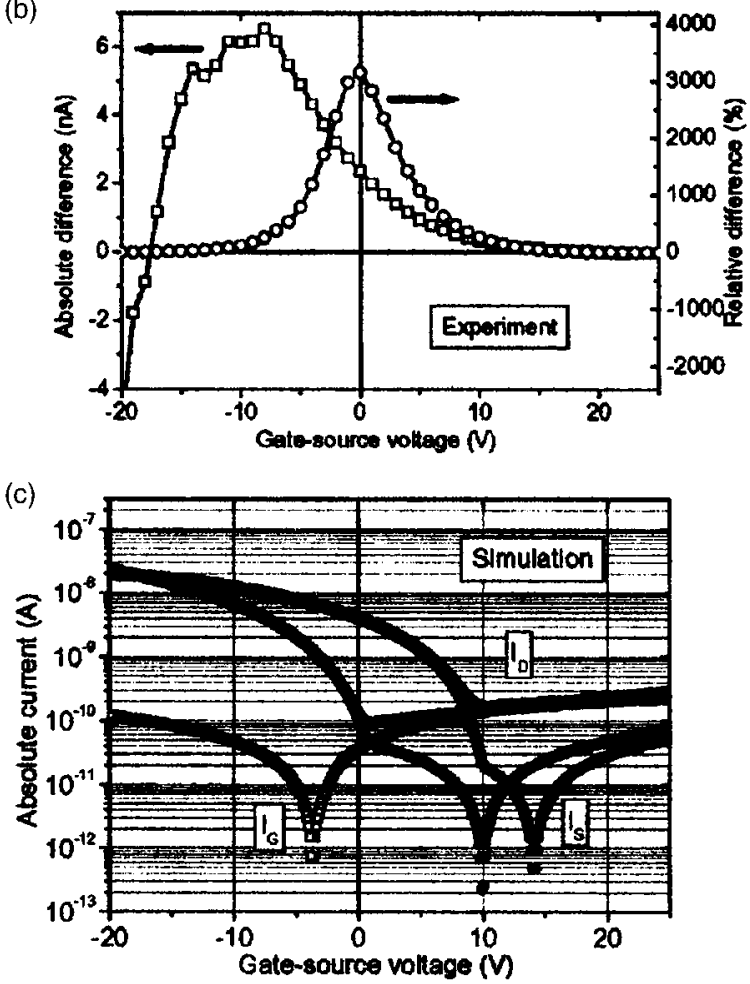

(d)

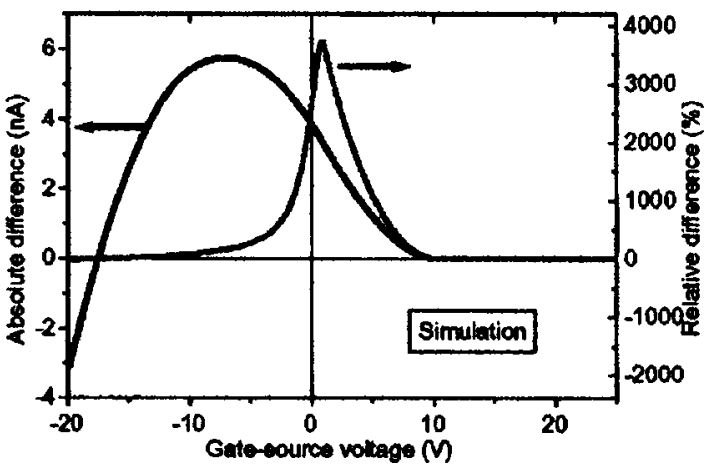

FIG. 4. (a) Measured transfer characteristics of a TFT with interdigitated source/drain contacts and a channel length of $10 \mu \mathrm{m}$, showing drain current, source current, and gate current; (b) measured relative $(\bigcirc)$ and absolute $(\square)$ changes in drain current between pressure and no-pressure states; (c) simulated transfer characteristic of the same device; and (d) simulated relative (gray) and absolute (black) changes.

$\left.-V_{\mathrm{TH}}\right)$ and $V_{\mathrm{GS}}<V_{\mathrm{TH}}$. The theoretical $I_{D}$ vs $V_{\mathrm{GS}}$ relationship is nearly linear in this range of gate-source voltages, but for organic semiconductors it is rather exponential. This inaccuracy of the model is insignificant for the discussion of the 

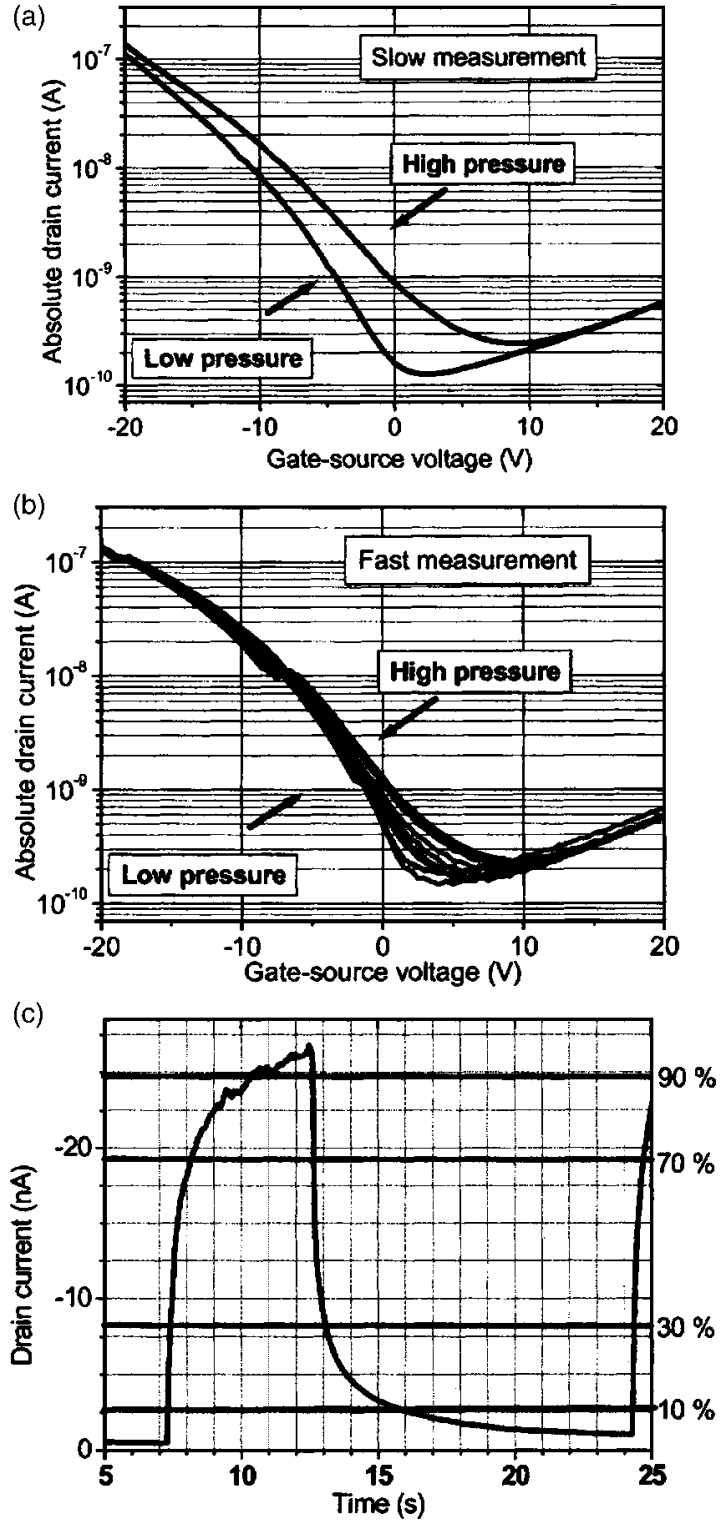

FIG. 5. (a) "Slow" measurement showing drain current in two steady states at $V_{\mathrm{DS}}=-20 \mathrm{~V}$, (b) "fast" measurement showing shifting drain current between the two steady states at $V_{\mathrm{DS}}=-20 \mathrm{~V}$, and (c) time dependence of drain current at $V_{\mathrm{GS}}=10 \mathrm{~V}$ and $V_{\mathrm{DS}}=-20 \mathrm{~V}$ when applying and removing pressure.

observed pressure effect since it can be corrected by fitting the model equation only.

The good agreement between our model and the experimental results is evident from Figs. 4(b) and 4(d), which show the pressure-induced relative and absolute changes in drain current, suggesting that our simulations provide a useful analysis of the mechanism causing the observed pressureinduced change in drain current. To achieve this agreement between simulation and experiment we modified the threshold voltage (VTO), mobility (UO), source resistance (RS), and drain resistance (RD) SPICE-model parameters as a response to applied mechanical pressure. We therefore conclude that the force-induced change in drain and source currents is due to the distribution and activity of trap states at or near the semiconductor/dielectric interface. This assumption

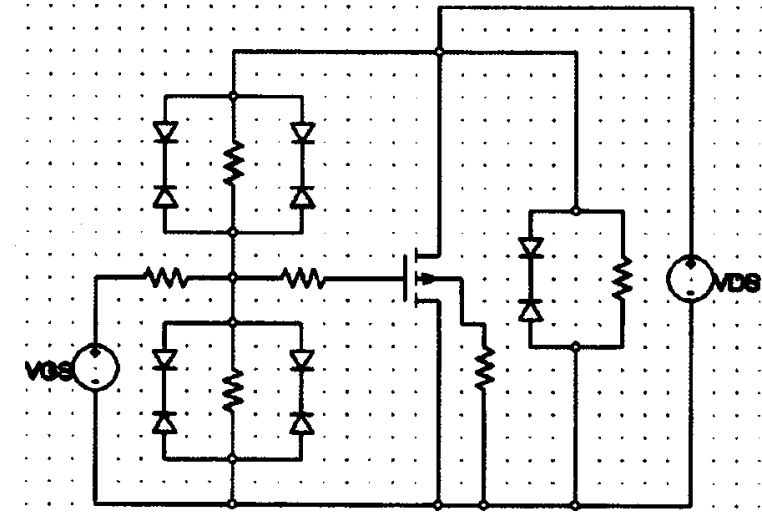

FIG. 6. Schematic of the extended SPICE $p$-MOSFET model used to simulate the leakage currents.

is further supported by the fact that we do not observe a change in current when the pressure is applied only to the channel region but not to the source and drain contacts [see Fig. 1(c)]. This conclusion is in line with previous reports on the importance of properly contacting pentacene. ${ }^{13,15}$

\section{CONCLUSIONS}

In summary, we have shown that organic thin-film transistors are influenced by direct mechanical pressure. By simulating the results we have shown that mechanical pressure applied directly to the TFT (including the contact regions) results in changes in carrier mobility, threshold voltage, and contact resistances. We have shown a dependence on materials and interfaces as the effect was only measured when contact regions were affected. The origin of the observed effect is not completely clear yet; we believe that trapped charges are responsible. However, the trapping mechanism and the nature of the traps are still under discussion.

${ }^{1}$ C. D. Sheraw et al., Appl. Phys. Lett. 80, 1088 (2002).

${ }^{2}$ B. K. Crone, A. Dodabalapur, R. Sarpeshkar, A. Gelperin, H. E. Katz, and Z. Bao, J. Appl. Phys. 91, 10140 (2002).

${ }^{3}$ T.-T. Zhu, J. T. Mason, R. Diekmann, and G. G. Malliaras, Appl. Phys. Lett. 81, 4643 (2002)

${ }^{4}$ J. A. Rogers et al., Proc. Natl. Acad. Sci. U.S.A. 98, 9 (2001).

${ }^{5}$ T. W. Kelley, L. D. Boardman, T. D. Dunbar, D. V. Muyres, M. J. Pellerite, and T. P. Smith, J. Phys. Chem. B 107, 24 (2003).

${ }^{6}$ G. B. Blanchet, Y.-L. Loo, J. A. Rogers, F. Gao, and C. R. Fincher, Appl. Phys. Lett. 82, 463 (2003).

U. Zschieschang, H. Klauk, M. Halik, G. Schmid, and C. Dehm, Adv. Mater. (Weinheim, Ger.) 15, 1147 (2003).

${ }^{8}$ M. Halik et al., Nature (London) 431, 963 (2004).

${ }^{9}$ T. Someya and T. Sakurai, Tech. Dig. - Int. Electron Devices Meet. 2003, 203.

${ }^{10}$ H. Klauk, M. Halik, U. Zschieschang, F. Eder, and G. Schmid, Appl. Phys. Lett. 82, 4175 (2003).

${ }^{11}$ M. Halik, H. Klauk, U. Zschieschang, T. Kriem, G. Schmid, and W. Radlik, Appl. Phys. Lett. 81, 289 (2002).

${ }^{12}$ R. Brederlow et al., Dig. Tech. Pap.-IEEE Int. Solid-State Circuits Conf. 2003, 378.

${ }^{13}$ P. V. Necliudov, M. S. Shur, D. J. Gundlach, and T. N. Jackson, J. Appl. Phys. 88, 6594 (2000).

${ }^{14}$ Explanation can be found in every SPICE documentation on MOSFETs.

${ }^{15}$ J. A. Nichols, D. J. Gundlach, and T. N. Jackson, Appl. Phys. Lett. 83, 2366 (2003). 